\title{
Further cosiderations of isoamylase pitfalls
}

In reference to a recent study by Hoeke $e t$ al.$^{1}$ of Joseph's saccharogenic method for the detection of isoamylase bands after agar electrophoresis ${ }^{2}$ : Hoeke $e t$ al ${ }^{1}{ }^{1}$ conclude that "The location of our glucose band is similar to that of the 'liver amylase" in the study of Joseph et al.", and that "...little if any amelioration of the interpretation of an increased serum amylase is to be expected from the method proposed by Joseph et al.".

As Hoeke $e t$ al. have shown ${ }^{1}$, glucose, when present in relatively high concentrations, can exhibit bands in a similar position to those due to amylases, with the electrophoretic buffer and conditions employed by Joseph et al. ${ }^{2}$. However, it has been demonstrated by means of total levels done on agar segments along the migration path, and by comparisons to an amyloclastic method, that the saccharogenic method is effective in detecting amylase activity ${ }^{2}$.

The results obtained by Joseph et al. were described as preliminary, and the only claim made was that the method "...may yield useful clinical information...".

I would like to suggest that, with proper blanking (i.e., omission of maltase and starch), which should always be done in any case, the method may still yield useful information. Any bands due to glucose can readily be distinguished from those due to amylase by the use of such blanks.

It is quite possible that, with further study, the preliminary results obtained by Joseph $e t$ al. can be improved in regard to resolution and sensitivity. It is also possible that the difference in migration between glucose and isoamylase bands can be increased by the use of different electrophoretic buffers.

Interference due to proteins in the amyloclastic methods of isomylase detection cannot be as readily blanked out as can interferences due to glucose in the saccharogenic method. Consequently, I feel it would be unfortunate if the procedure of Joseph et al. were completely dismissed, as Hoeke et al. would appear to suggest.

Investigations of isoamylases have been supported by a grant from the U.S. Public Health Services.

Departments of Pathology and Biochemistry,

N. RESSLER University of Michigan, Ann Arbor, Mich. (U.S.A.)

I J. O. O. Hoeke, C. Voorrips and J. De Wael, Clin. Chim. Acta, i6 (1967) I7I.

2 R. R. Joseph, E. Olivero ANd N. Kessler, Gastroenterology, 5 I (I966) 377.

Received May 3rd, 1967 